Témoigner Témoigner. Entre histoire et mémoire

Getuigen Revue pluridisciplinaire de la Fondation Auschwitz

$123 \mid 2016$

Traduire le témoignage

\title{
Saul Fia / Son Of Saul / Le fils de Saul,
} paroles de morts

Saul Fia / Son Of Saul, de doden spreken

\section{Erik Machielsen}

\section{(2) OpenEdition \\ 1 Journals}

Édition électronique

URL : https://journals.openedition.org/temoigner/5038

DOI : 10.4000/temoigner.5038

ISSN : 2506-6390

\section{Éditeur :}

Éditions du Centre d'études et de documentation Mémoire d'Auschwitz, Éditions Kimé

\section{Édition imprimée}

Date de publication : 1 octobre 2016

Pagination : 9-12

ISBN : 987 2-9600926-4-6

ISSN : 2031-4183

\section{Référence électronique}

Erik Machielsen, "Saul Fia / Son Of Saul / Le fils de Saul, paroles de morts », Témoigner. Entre histoire et mémoire [En ligne], 123 | 2016, mis en ligne le 02 novembre 2021, consulté le 04 novembre 2021. URL : http://journals.openedition.org/temoigner/5038; DOI : https://doi.org/10.4000/temoigner.5038 


\section{SAUL FIA / SON OF SAUL / LE FILS DE SAUL PAROLES DE MORTS}

CINÉMA Est-il possible de traduire l'horreur d'Auschwitz par le biais du cinéma de fiction?

Oui. La preuve, douloureuse et puissante, par Son of Saul.

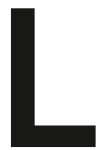

a mâchoire serrée, le visage fermé cadenassé dans le cadre étriqué de l'image... Nous sommes en octobre

1944, à Auschwitz-Birkenau et ce visage est celui de Saul Ausländer, un Sonderkommando, unité regroupant des détenus juifs forcés d'assister les nazis dans leur basse besogne, assurant jour et nuitla cruelle intendance qui forgera la Shoah : canaliser les troupeaux humains nus vers les chambres à gaz, récupérer les cadavres, les brûler et disperser leurs cendres. Après quoi, eux aussi mourront.

Ce jour-là, parmi les corps qui s’amoncellent, Saul croit reconnaitre celui de son fils. Allant à l'encontre de toute sécurité, il n’a plus qu’une obsession : assurer à l'adolescent une sépulture digne et l'enterrer selon les rites de sa religion. Inhibé, traumatisé, Saul devient imperméableà ce quil'entoure, perd la notion des choses, se retranche dans son monde. Il se bâtit une prison dont il a jeté la clé. Une prison dans laquelle il nous emmure.

Bien qu'abasourdis par le rythme et abrutis par la bande son du film, on s'interroge sur certaines situations, sur l'attitude de cet homme qui n'hésite pas, de manière quasi suicidaire, à mettre ses camarades de misère en péril. On se demande même si la dépouille qu'il véhicule est bien celle de son fils... Le chagrin de la perte de cet enfant et le désespoir qui s'en est suivi l'auraient rendu fou. C'est une explication, un doute que le réalisateur introduit dans la séquence finale.

Ici, c'est clair, on est loin du divertissement, de l’aventure, du cinéma spectacle à la Spielberg aussi louables qu'aient été ses intentions. Résigné, abattu, Saul est une victime tétanisée par la souffrance. Dès le début, il n'est plus un homme, mais un zombie, un automate errant parmi un chaos permanent. Voilà pourquoi il n'a plus peur de rien : il a atteint un point de non-retour où la seule chose qui importe est la promesse qu'il s'est faite. Insensible au reste, inhibé dans sa sphère, inconscient des conséquences de son attitude, injuste dans ses soupçons, il s'accroche désespérément à des illusions, s'impose des œillères quand il rencontre un homme qui, pour sauver sa peau, se fait passer pour le rabbin qu'il n'est pas.

\section{LA VÉRITÉ PRIMÉE}

Grand prix du $68^{\mathrm{e}}$ Festival de Cannes, Golden Globe du meilleur film étranger en 2016, Son of Saul a récolté plus d'une quarantaine de récompenses. La Belgique n’a pas été en reste qui lui a décerné le Grand Prix de l'Union de la Presse Cinématographique Belge (UPCB) en janvier dernier.Et ce, un mois avant la consécration suprême: Oscar du meilleur film étranger à Hollywood. À cet égard, on observera que c'est le sixième film traitant de la Shoah à l'avoir obtenu. Les cinq précédents étant : Obchod na korze de Ján Kadár et Elmar Klos (1965), Il giardino dei Finzi Contini de Vittorio De Sica (1971), La vita è bella de Roberto Begnini (1998), Die Fälscher de Stefan Ruzowitzky (2007) et Ida de Pawel Pawlikowski (2014). Il convient également de citer un nom sur lequel on ne peut faire l'impasse, celui de Tamás Zányi, ingénieur du son qui a obtenu au Festival •.• 
œ. de Cannes le Prix Vulcain de l'artiste technicien pour la contribution exceptionnelle duson à la narration du film». On ne peut mieux dire.

ITINÉRAIRE D'UN HONGROIS GÂTÉ

En cette année 2016, László Nemes comptabilise 39 années. Il n’était donc pas né au moment des faits, Saul une partie de sa fanille a péri à Auschwitz. Son of divest son prenier long métrage. En 2007, il remporte divers prix á Lisbonne, Angers, Bibao pour Turelem, ù court métrage de 14 mintes. Celui quil le couronn a Budapest est accompagné dun commentaire: «pour qui préfigure parfaitement la forme que prendra Le quis pre Sal hut ans plus tard : cela se pre peja de fils de Saul huit ans plus tard. cela se passe deja dan un camp, mais surtout, c'est capte par une camera qui delle tou sur flo visage d une jeune fenme - autour elle tout est flou. Aucun dialogue, rien que du son Kubrick, Tarkovski, Bergman et Malick, Nemes fait Kubrick, Tarkovski, Bergnan et Malick, Nemes fait montre d'une patte qui d'enble le distingue de ses confrères.

L'ÂME DE SAUL

Une des forces du film - et commercialement parlant cela aurait pu être une faiblesse - est qu'on n'en connait pas les interprètes. Avantage : on voit le personnage et non l'acteur qui l'incarne.

Et cet acteur est Géza Röhrig. L'abandon, la solitude, l'isolement, il les a connus : abandonné par sa mère, elevé dans un orphelinat, à lâge de 4 ans il per son père et est adopté, à 12 ans, par une famille juive cì une viste ductéch juive. Cette visite ne devit se mat a jou juive. Cette visite ne devait durer qu'un jour. Marqué parce quili voit,

Sorti diplômé de l'École supérieure d'art dramatique et cinématographique de Budapest où un de ses professeurs fut Istvan Szabó, (le réalisateur du pis qui, wurtout, fut, en 1982, le premi Brilm mais qui, surtout, fut, en 1982, le premier film honLeis à remporter lOscar du meilleur film étranger. cesecondétant....Son ofSaun, cest cependanten tan quécrivain et plus encore en tant que poète, que Rörig e distingue, publiant sa première œuvre en 1995: des sétait jusquelà limitće à deux ou tris appartions a la télévision à la fin des années 1980. László Nemes a rencontré Röhrig à New York (où il vit) dans les années 2000. Ils se sont découvert des interêts comfilm hiln. Et on inagine le danger qua pu représenter cette expérience - sa prestation a elle aussi été primée à de teur n'en sort pas indemne.

A GENÈSE

C'est en 2011, à Paris (il parle couramment le français) et en compagnie de Clara Royer, que László nariste françise d'origine hongroise, Clara Royer s'est fait lequel l'héroï avec un premier roman, Csillag, dans lequel lhèroine, en quête didentité, entreprend des zmann, So of Saul s'ins. Non de zmann, Son of Saul s'inspire de faits réels consignés lectif publie 2005 qui lacendre, un ouvrage collectif puble en 2005 qui reprend trois des cinq ecrits de Sonderkommandos, enfouis dens le sol de Birkenau

Le fait de se concentrer sur un seul personnage fait refeérence à un autre film de guerre, soviétique celui-là, Idi i Smotri / Requiem pour un massacre dElem Klimov, tourne en 1985 et composé lui auss de longs plans séquences, de bruitages plus que de dialogues, de cris, de pleurs qui entretiennent la peur langoisse, Timminenc ant peut frapper

nnfin, Son of Saul résulte aussi d'un travail en profondeur étalé sur quatre ans. Le temps nécessaire pris par Nemes et Royer pour se documenter et renconGidéon Greif, détenteur de témoignages de resaélien Gidéon Greif, détenteur de témoignages de rescapé

DILEMME

a différence suscite souvent le rejet et cette différence réside ici dans la forme. Voilà pourquoi, un mode d'emploin'est pas la forne. Volla pourqui, un mode demploinest pas superfuavant daborder Son of Saul. Ony est confronté un langage qui conditionne - si on en ignótentestré que tantril voit par le personnage, la caméra ne filme que ce qu il voit et rour latention. Sa douloú layant séni forgé une carapace au point de basculer dans le lisateur la floute.



DILEMME 2

Son of Saul est aussi le type même de film que l'o : Car que ce soit en France en Isräll, en Aleme Car que ce soit en France, en Israel, en Allemagne ou en Autriche, nulle part on na voulu le financer : trop ien que remarquablement éclairé, l'esthétique et la Bien que remarquablenent éclairé, Iesthétique et a l'ima en elle est complétée par une baude. Quant a limage, elle est completee par une bande son tout aussi - si pas plus - explicite. Et bien que le récit soit qui répond au éntion, on ne peut sen échapper. Ce quir repond au sentiment du reallsateur : revenir en ar ma à Les morts láunir le Cela précisé, sice dernier a tout de même pu film, cest principanemt au Fond du Film hongrois culil cest principa

Certes le pari de Nemes est gagné, la multitude de récompenses en atteste. Mais la plupart ont été décernées par des jurys composés délites et de spécialistes qúplics peuvent niter la majorité silencieuse à se den treuvera nont pas partie. Parnicette dernière, sen trouvera donc toujours pour prétendre que ces prix furent accordés pour des motifs autres que cinématonitaires ou même politiques. À des raisons humaqu’un filmà à point réfléchi
Parce qu'il a mis du temps à se construire, il est normal qu'il prenne du temps à être compris, à être perçu pour les bonnes raisons, celles pour lesquelles il a éte conçu. On peut donc le trouver détestable, raté même succédé à la déroute qu’a suscité sa forme.

DILEMME 3

Bref, que certains n'adhèrent pas à cette forme c'est leur droit. Mais personne ne sera indifféren u fond : l'enfer d'Auschwitz-Birkenau. À cet égard le film est exemplaire grâce à un travail fouillé de la bande sonore : aucune musique, seulement le brouhaha de cris, de portes qui grincent, de coups de feu qu claquent. Tout cela contribue à créer une atmosphère pit-il en arriver lá échét ét dement demeure en permanence cloîtré dans le gros plan pour

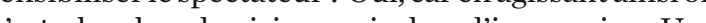
n'est plus dans la vision mais dans limmersion. Une déconsille à le film aux personnes tropém êra jusqua déconseillor lefilm auxpersons Ce point précise l'excis nuit e

Ce point précisé, l'excès nuit en tout. Cheville la caméra au visage du protagoniste et pratiquer un s'ériger en la Dardenne puissance 100 ne devraient pas à la case «On en a vu un, on les a tous vus.» Dès lors, 
- c'est avec une certaine fébrilité qu'on attend son prochain film, lequel sera encore issu de sa collaboration avec Clara Royer. On n'en connait pas le titre, mais on sait déjà que ce sera un thriller, à nouveau immersif et construit cette fois autour d'une femme.

\section{LE FILS DE LANZMANN}

Dès que se projette une œuvre ayant traità la Shoah, la comparaison avec le mètre étalon qu'est Shoah, documentaire fleuve de plus de dix heures sorti en 1985, monte directementàl'esprit. Et Claude Lanzmann, son réalisateur, est régulièrement sollicité pour savoir ce qu'il en pense. Sans jamais les dénigrer - la démarche de s'attarder sur la Shoah appelle déjà au respect - il n’a pujusque-là s'empêcher, même dans le cas de Spielberg et sa Liste de Schindler, de considérer que le drame de la Shoah servait trop souvent de décor à des réalisateurs qui biaisaient le sujet en y apposant leur empreinte. On voyait les camps mais on n’y était pas. Jusqu’à ce qu’il visionne Son of Saul lors du dernier Festival de Cannes. Il s'y était rendu sur base de la réputation naissante du film mais avec un enthousiasme mesuré. Il en est ressorti conquis en déclarant : «C'est dans le cinéma de fiction, le seul où je trouve autant de vérité que dans Shoah. Je le considère comme son égal. » Et d’adouber son réalisateur en le désignant, artistiquement, comme son fils.
Savoir qu'un homme de l'ampleur de Lanzmann crédite le film n'est donc pas inutile pour inciter le grand public à franchir le pas et à aller le voir.

Et prétendre que Son of Saul a, comme Shoah, valeur emblématique n'est du coup pas abusif. Le devoir de mémoire est le même, mais le registre diffère : de contemplatif - le terme peut paraître maladroit - on est passé à l'immersif. Et le fait que le second ait été pensé et réalisé par des héritiers de la Shoah, et non par ses contemporains, n'en a que plus de résonance.

\section{À DÉCOUVRIR ABSOLUMENT}

Beaucoup n'ont pas encore tiré les leçons du génocide et la barbarie existe encore. En cela, Son of Saul est salutaire, car il lui fait rempart. Allez le voir pour que nos enfants ne connaissent «Plus jamais ça ». Et de préférence dans une salle bien sonorisée. Le voir en DVD dans un salon n'a aucun sens. I

Erik Machielsen

\section{Plus d'infos}

$\rightarrow$ Saul fia / Son of Saul / Le fils de Saul de László Nemes (2015).

Avec : Géza Röhrig (Saul Ausländer), Levente Molnár (Abraham Warszawski), Urs Rechn (Oberkapo Biederman) 107 minutes.

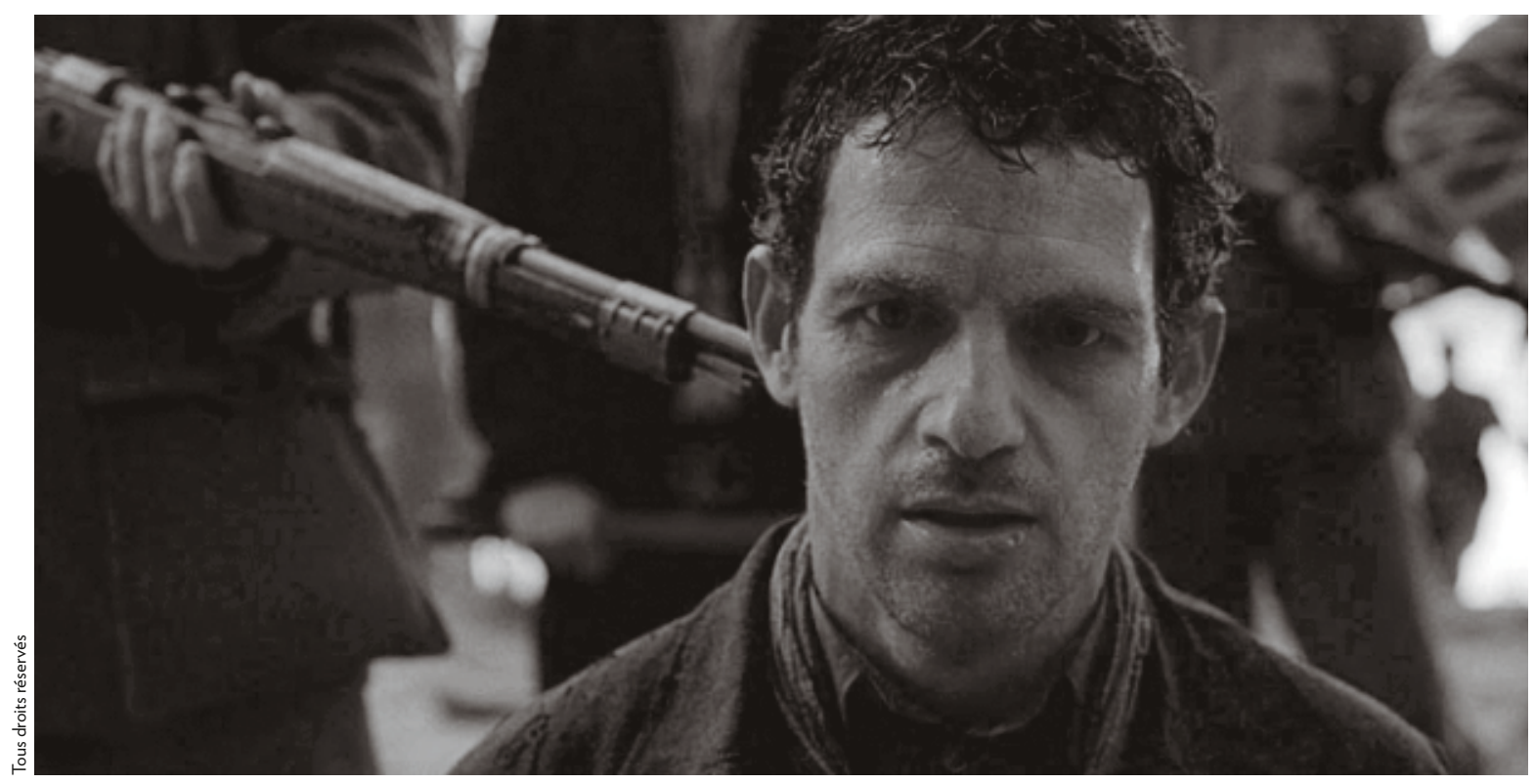

